\title{
Prevalência e fatores associados à síndrome do imobilismo em pacientes oncológicos internados
}

\author{
Prevalence and factors associated with immobilism syndrome in hospitalized cancer \\ patients
}
Prevalencia y factores asociados con el síndrome de inmovilismo en pacientes con cáncer hospitalizado

Flávia Maria Ribeiro Vital ${ }^{1 *}$, Camila Bertini Martins ${ }^{2}$.

\begin{abstract}
RESUMO
Objetivo: Determinar a prevalência e gravidade da síndrome do imobilismo (SI) e sua associação com sintomatologias, eventos adversos e perdas funcionais em pacientes com câncer internados. Métodos: Foi realizado um estudo transversal analítico em pacientes internados em instituição terciária de oncologia. Como ferramentas de análises dos desfechos propostos utilizou-se a classificação de gravidade da SI, a Escala Visual Analógica (EVA), o Pictograma de fadiga (PF) e a Classificação Internacional de Funcionalidade (CIF). O nível de significância utilizado nas análises de associação das variáveis foi de $5 \%$. Resultados: A prevalência da SI em pacientes com câncer internados foi de $52 \%[0,44 ; 0,60]$ e $15 \%[0,09 ; 0,58]$ para SI clinicamente relevante. A SI demonstrou associação significativa com fraqueza muscular $(\mathrm{OR}=5,17 ; \mathrm{IC} 95 \%=[1,93 ; 14,60])$, alta intensidade de estresse (OR=5,5; IC95\% [1,96;17,50]) e fadiga de moderada (OR=3,56;IC95\%[1,57;8,58]) e alta intensidade (OR=7,75;IC95\%=[1,92; 52,20]). A gravidade progressiva da SI se correlacionou com a progressiva intensidade de fadiga e estresse pela EVA, intensidade e impacto da fadiga nas atividades diárias pelo PF e, também, na capacidade funcional pela CIF. Conclusão: A SI tem uma prevalência significativa em pacientes com câncer internados e sua presença e gravidade se correlacionam com fadiga, estresse e perdas funcionais.
\end{abstract}

Palavras-chaves: Limitação da mobilidade, Prevalência, Neoplasias, Unidades de internação.

\begin{abstract}
Objective: To determine the prevalence and severity of immobilism syndrome (IS) and its association with symptoms, adverse events and functional losses in hospitalized cancer patients. Methods: A cross-sectional analytical study was conducted in patients hospitalized in tertiary oncology institution. As tools for analysis of the proposed outcomes, we used the severity classification of IS, the Visual Analog Scale (VAS), the Fatigue Pictogram (FP) and the International Classification of Functionality (ICF). The level of significance used in the analysis of the association of the variables was $5 \%$. Results: The prevalence of IS in hospitalized cancer patients was $52 \%[0.44 ; 0.60]$ and $15 \%[0.09 ; 0.58]$ for clinically relevant IS. IS showed significant association with muscle weakness(OR=5.17;Cl95\%=[1.93;14.60]), high stress intensity $(\mathrm{OR}=5.5 ; \mathrm{Cl} 95 \%[1.96 ; 17.50])$ and moderate $(\mathrm{OR}=3.56 ; \mathrm{Cl} 95 \%[1.57 ; 8.58])$ and high intensity fatigue $(\mathrm{OR}=7.75 ; \mathrm{Cl} 95 \%=[1.92 ; 52.20])$. The progressive severity of IS was correlated with the progressive intensity of fatigue and stress to VAS, intensity and impact of fatigue on daily activities by FP and also in functional capacity by ICF. Conclusion: IS has a significant prevalence in hospitalized cancer patients and its presence and severity correlate with fatigue, stress and functional losses.
\end{abstract}

Keywords: Mobility limitation, Prevalence, Neoplasms, Inpatient care units.

\section{RESUMEN}

Objetivo: Determinar la prevalencia y gravedad del síndrome de inmovilización (SI) y su asociación con síntomas, eventos adversos y pérdidas funcionales en pacientes con cáncer hospitalizado. Métodos: Se realizó un estudio analítico transversal en pacientes hospitalizados en instituciones oncológicas terciarias. Como herramientas para el análisis de los resultados propuestos, utilizamos la clasificación de gravedad de

1 Vital Knowledge, Muriaé - MG. *E-mail: flavia_vital2000@yahoo.com.br

2 Universidade Federal de São Paulo (UNIFESP), São Paulo - SP. 
SI, la Escala Analógica Visual (EAV), el Pictograma de Fatiga (PF) y la Clasificación Internacional de Funcionalidad (CIF). El nivel de significancia utilizado en el análisis de la asociación de las variables fue del $5 \%$. Resultados: La prevalencia de SI en pacientes con cáncer hospitalizado fue del $52 \%[0,44 ; 0,60]$ y del $15 \%[0,09 ; 0,58]$ para la SI clínicamente relevante. SI mostró una asociación significativa con la debilidad muscular (OR=5,17;IC95\%=[1,93;14,60]), alta intensidad de tensión (OR-5,5; IC95\% [1.96;17.50]) y fatiga moderada (OR-3.56;IC95\%[1.57;8.58]) y alta intensidad (OR-7.75;IC95\%-[1.92; 52.20]). La gravedad progresiva del SI se correlacionó con la intensidad progresiva de la fatiga y el estrés debido al EAV, la intensidad y el impacto de la fatiga en las actividades diarias por PF y también en la capacidad funcional por CIF. Conclusión: SI tiene una prevalencia significativa en pacientes con cáncer hospitalizado y su presencia y gravedad se correlacionan con fatiga, estrés y pérdidas funcionales.

Palabras-clave: Limitación de la movilidad, Prevalencia, Neoplasias, Unidades de internación.

\section{INTRODUÇÃO}

O repouso absoluto durante uma internação vem sendo questionado desde a década de 40 do século passado e progressivamente foi-se entendendo e incentivando a mobilização precoce em pacientes cardiopatas clínicos e cirúrgicos (CAO J, et al., 2021; KEHLER DS, et al., 2019). Somente neste século é que esta intervenção foi expandida para pacientes submetidos a outras cirurgias de grande porte e em pacientes críticos, onde muito se tem discutido o impacto da imobilidade e a efetividade e segurança da mobilização precoce (SANTOS C, et al., 2016; HERMANS G, et al., 2014). Todavia ainda é escassa a literatura avaliando o impacto e frequência da redução da mobilidade desenvolvida durante uma internação hospitalar em contextos diferentes da geriatria (ESPÍNDOLA R, et al., 2020).

É reconhecido que o repouso ou a falta de atividade física podem resultar em um grave descondicionamento, redução da funcionalidade física e vários eventos adversos em diferentes sistemas orgânicos (CAO J, et al., 2021; GUEDES LPCM, et al., 2018; KEHLER DS, et al., 2019; SAUNDERS CB, 2015). A imobilidade pode reduzir a massa muscular pela metade em menos de duas semanas provocando uma perda de força muscular de 4-5\% por semana (GARCÍA-PEREZ L, et al., 2011; LUM HD, et al., 2012). A redução da mobilidade desenvolvida durante uma internação hospitalar pode ser causa de incapacidade funcional por até um ano, tornando os indivíduos inábeis para o retorno ao trabalho devido à fraqueza e fadiga persistentes (ZHANG L, et al., 2019).

Embora a mobilização precoce seja uma intervenção que tenha seus efeitos comprovados em vários estudos que validam o projeto Enhanced Recovery After Surgery (ERAS) ou mesmo nas unidades de terapia intensiva (UTIs), pouco se discute as causas que levam a necessidade desta intervenção, como a síndrome do imobilismo (SI), ou mesmo a frequência com que ela pode estar presente em determinados contextos de saúde como uma internação hospitalar (BOND-SMITH G, et al., 2016; CHEN S, et al., 2011; CONNOLLY B, et al., 2016; FOSSAT G, et al., 2018; KANG SH, et al., 2018; MACHADO ADS, et al., 2017; SHIDA D, et al., 2017; ZHANG L, et al., 2019).

A SI foi definida como "o conjunto de sinais e sintomas relacionados à mobilidade que são manifestos pelas disfunções orgânicas geradas devido à redução de nutrientes e oxigênio que são carreados pelo sistema cardiovascular cujo potencial funcional tem influência direta do nível de atividade física", ou seja, um menor nível de atividade física, expressa pela redução progressiva da mobilidade (sedentarismo, mobilidade reduzida, restrição no leito e imobilidade no leito), tende a hipoativar o sistema cardiovascular, uma vez que o sistema musculoesquelético é um dos maiores consumidores de oxigênio para execução das suas funções básicas (VITAL FMR, 2017).

Desta forma, menos oxigênio e nutrientes tendem a serem distribuídos, também, a outras células do organismo, as quais, se estiverem no limiar da sua função, podem manifestar sinais e sintomas que caracterizariam a síndrome do imobilismo e tenderia a levar esse organismo a uma redução ainda maior da sua mobilidade (PLAPLER PG, et al., 2021; VITAL FMR, 2017).

Adicionalmente, os tratamentos oncológicos, sejam eles cirurgia, radioterapia ou quimioterapia, na tentativa de eliminar as células malignas, acabam por afetar células sadias também, com isso provocam 
efeitos adversos como dor, fadiga e dispneia, os quais são autolimitantes e tendem a reduzir a mobilidade dos pacientes e consequentemente tendem a enquadrar os pacientes na SI e aumentar o risco de gravidade dela, reduzindo assim a capacidade funcional e possivelmente a qualidade de vida dos pacientes, com possíveis impactos, também, na sobrevida (SILVER JK, et al., 2013).

Várias complicações podem surgir ou se agravarem com a redução da mobilidade durante uma internação, como: pneumonia, atelectasia, lesões por pressão, aumento da resistência à insulina, ansiedade, depressão, delirium, constipação, hipotensão postural, fadiga, fraqueza muscular, encurtamentos de músculos, tendões, anquiloses articulares, osteoporose, descondicionamento cardiovascular, trombose venosa profunda (TVP) e/ou tromboembolia pulmonar (TEP) (CAO J, et al., 2021; GUEDES LPCM, et al., 2018). Pelo Escore de Pádua, se um paciente com câncer ficar restrito ao leito por mais de 3 dias, ele passa a ter um risco de $11 \%$ de ter um evento tromboembólico. A TEP é a principal causa da morte de paciente com câncer. Mesmo em pacientes que desenvolvem uma TVP e estão anticoagulados, a deambulação precoce demonstrou ser segura e pode contribuir no alívio da dor (IZCOVICH A, et al., 2016; LIU Z, et al., 2015).

Pacientes que já se internam com algum grau de déficit de mobilidade, fraqueza ou fadiga ou aqueles que têm o prognóstico de necessidade de internação maior do que três dias, mas que estão com estabilidade clínica, em especial hemodinâmica e respiratória, estando ele em ventilação espontânea ou mecânica, deveriam receber avaliação e intervenção de fisioterapia para prevenir a SI, considerando a segurança para tal (PLAPLER PG, et al., 2021). Um estudo coorte observacional que acompanhou 30 pacientes com tumores sólidos e 30 pacientes com tumores hematológicos, onde todos recebiam reabilitação física, observou-se uma melhora de 13\% na independência funcional ao longo de 10 dias de internação (GUO Y, et al., 2011).

A Sociedade Brasileira de Cardiologia afirma em seus consensos de cardio-oncologia, que quanto menor a mobilidade maior a perda de função orgânica, da capacidade funcional e consequentemente da qualidade de vida e que a redução de atividades pode levar ao acúmulo intracelular de antraciclina e toxicidade celular (KALIL FILHO R, et al., 2011; HAJJAR LA, et al., 2020).

Portanto, objetivamos identificar a prevalência e gravidade da SI presente em pacientes oncológicos internados, assim como o perfil dos pacientes e os momentos da doença câncer e/ou tratamento com maior risco de desenvolver a SI, além de possíveis associações desta síndrome com quadros clínicos e funcionais que possam ajudar a identificar o potencial de risco de manifestação da SI, para assim, termos um parâmetro facilitador para identificação precoce do problema e um melhor direcionamento das intervenções, a base de exercícios físicos, que possam reduzir o impacto clínicos, econômicos e sociais da SI.

\section{MÉTODOS}

Foi realizado um estudo observacional do tipo transversal analítico que incluiu pacientes adultos atendidos no mês de fevereiro ou junho de 2017 que já possuíam o diagnóstico de câncer e se encontravam internados em um hospital terciário especializado em oncologia. Foram excluídos pacientes com sequelas prévias ao câncer ou início do tratamento oncológico que afetavam a mobilidade, assim como aqueles com incapacidade para responder verbalmente ou por análise visual das escalas a sua percepção de dispneia, dor, fadiga, náusea e estresse.

A amostra estimada para este estudo foi de 96 pacientes considerando $50 \%$ a proporção de pacientes com SI presente na amostra total de pacientes investigados, erro tipo I de $5 \%$ e poder do teste de $10 \%$. Contudo como a amostra era facilmente disponível na instituição, foram analisados todos os pacientes presentes nas datas previstas, e alcançados pela equipe, para fortalecer a validade interna do estudo.

Após o adequado treinamento da equipe envolvida, foi realizada a coleta dos dados através de uma ficha padronizada. Na Figura 1 identificamos a classificação da gravidade da síndrome do imobilismo desenvolvida por Vital FMR (2017), a qual foi utilizada para mapear a intensidade da perda de mobilidade presente no momento da avaliação dos participantes. Esta classificação pode ser útil para identificar as limitações funcionais e nortear as condutas fisioterápicas necessárias em cada nível para alcançar a melhoria da mobilidade capaz de enquadrar o paciente em um grau menor de gravidade. 
Figura 1 - Classificação da gravidade da síndrome do imobilismo.

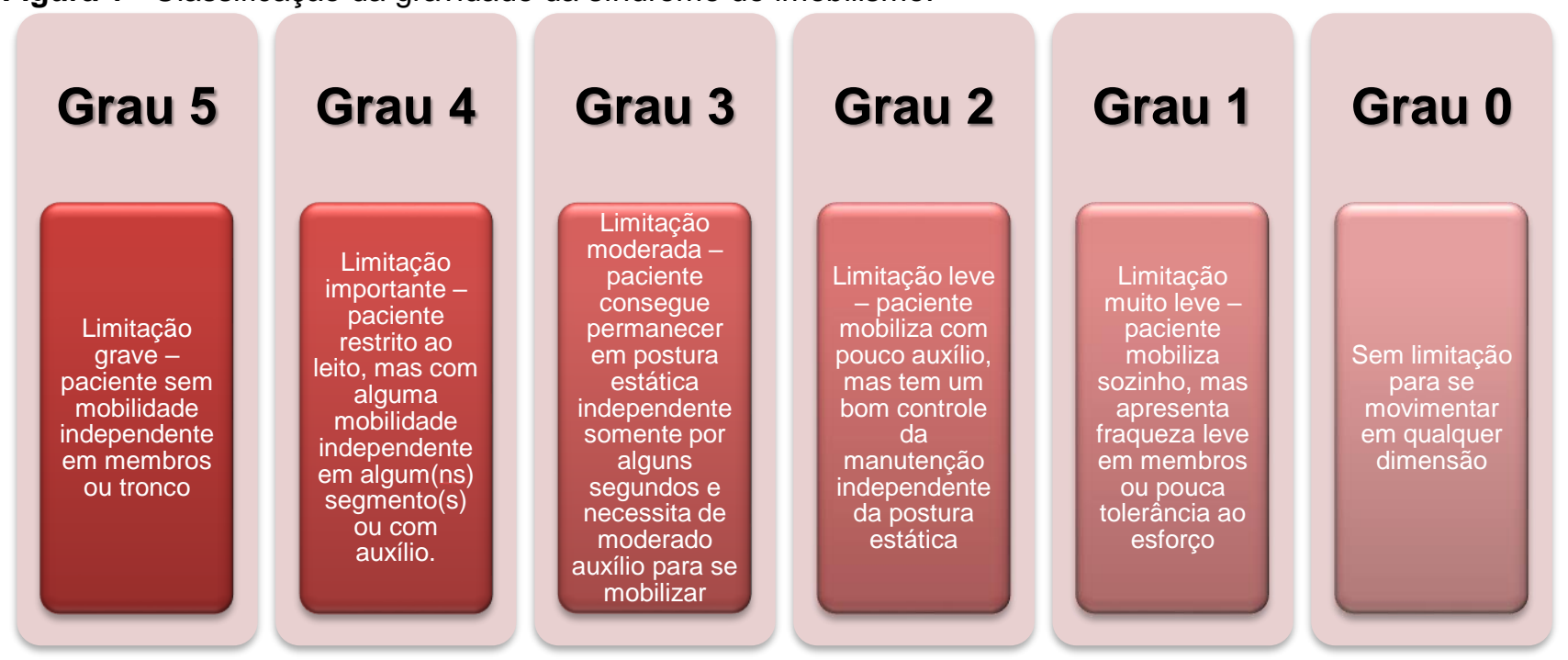

Fonte: Vital FMR e Martins CB, 2021; adaptado de: Vital FMR, 2017.

Foi utilizado, também, a escala visual analógica (EVA) para avaliação da intensidade dos sintomas dispneia de repouso, dor, náusea, estresse e fadiga, considerando 0 a ausência do sintoma e 10 o sintoma insuportável na percepção do paciente. O Pictograma de fadiga desenvolvido por Mota e colaboradores (MOTA DDCF, et al., 2009) foi utilizado para quantificar a intensidade de fadiga na última semana e o quanto a mesma impedia o paciente de fazer o que ele queria.

Adicionalmente utilizou-se da Classificação Internacional de Funcionalidade (CIF), elaborada pela Organização Mundial de Saúde, para mensurar a intensidade de deficiência para realizar funções como mudar a posição básica do corpo, andar distâncias curtas, realizar tarefas domésticas, relacionamentos sociais informais, realizar trabalho remunerado e função de executar a rotina (ORGANIZAÇÃO MUNDIAL DE SAÚDE, 2003). Os pacientes ou seus acompanhantes foram abordados em seus leitos de internação. Todos os pacientes incluídos foram abordados uma única vez, ainda que permanecessem internados por mais dias. Informações como diagnóstico do tumor primário, estadiamento da doença e complicações presentes foram coletadas do prontuário eletrônico dos pacientes incluídos.

Este estudo epidemiológico sobre a SI buscou responder sobre a prevalência e gravidade da SI na população de pacientes com câncer internados; assim como por estadio da doença (I a IV) e por subgrupos de cânceres (mama, próstata, cabeça e pescoço, tórax, ginecológico, sistema digestivo, hematológico, Sistema Nervoso Central (SNC), urológico, musculoesqueléticos ou pele), por tipo de tratamento recebido (quimioterapia, cirurgia, radioterapia e/ou suporte clínico) e por unidade de internação (enfermaria clínica, enfermaria cirúrgica, enfermaria de quimioterapia ou UTI), assim como sua correlação de gravidade progressiva da SI com a progressão de sintomas e perdas funcionais.

Foram aplicados o teste Qui-quadrado e Qui-quadrado via simulação de Monte Carlo para verificar a associação entre as variáveis categóricas e o desfecho (SI). O teste de Wilcoxon-Mann-Whitney foi utilizado para comparar as categorias (presença ou ausência) da SI com relação as variáveis ordinais. A razão de chances (OR) e seu respectivo intervalo de 95\% de confiança (IC 95\%) foram utilizados para medir as associações entre as variáveis do estudo e o desfecho (presença ou ausência da SI). E a correlação entre a gravidade da SI e variáveis sintomas, eventos adversos e funcionalidade foi avaliada pelo coeficiente de correlação de Spearman.

Os resultados foram descritos em termos de frequências absolutas (n), relativas (\%), mediana (med), intervalo interquartílico (IQR), razão de chances bruta (OR), intervalo de confiança de $95 \%$ (IC95\%) e p-valor. O nível de significância considerado foi de $5 \%$. Foi utilizado o software estatístico $R$ versão 3.6.3.

O presente estudo seguiu os aspectos éticos legais, respeitando as Diretrizes e Normas reguladoras de Pesquisas Científicas do Conselho Nacional de Saúde através da Resolução № 466 de 12 de dezembro de 
2012. O projeto de pesquisa foi enviado para apreciação do Comitê de Ética de pesquisa da FAMINAS, tendo sido aprovado com o número de parecer 1.843.904, sob CAAE: 62262516.8.0000.5105.

\section{RESULTADOS}

Foram incluídos 155 participantes, 60\% homens e 40\% mulheres. Em pacientes com câncer internados, a prevalência da SI foi de $52 \%[0,44 ; 0,60]$ e $15 \%[0,09 ; 0,58]$ para SI clinicamente relevante, ou seja, quando há redução da mobilidade que podem indicar a necessidade maior de reabilitação (classificação 3 a 5). Quando a SI estava presente, $44 \%$ dos participantes se enquadravam no grau 1, 27\% no grau 2, $12 \%$ no grau $3,9 \%$ no grau 4 e $7 \%$ no grau 5 . Não houve diferenças na amostra para presença ou gravidade da SI em relação à idade, sexo, raça ou estadiamento do tumor, mas foi possível observar que a depender do tipo de tratamento recebido ou do tipo de unidade de internação onde o paciente foi alocado pode ocorrer uma maior chance de se enquadrarem na SI. Já a depender do tipo de câncer por localização, pode ocorrer diferenças na prevalência associadas à gravidade da SI (Tabela 1).

Tabela 1 - Características da amostra em relação a presença e gravidade da SI.

\begin{tabular}{|c|c|c|c|c|c|c|c|c|c|c|}
\hline \multirow[t]{2}{*}{ Variável } & \multicolumn{2}{|c|}{$\begin{array}{c}\text { Total } \\
\mathrm{N}=155\end{array}$} & \multicolumn{2}{|c|}{$\begin{array}{c}\text { Ausente } \\
N=74\end{array}$} & \multicolumn{2}{|c|}{$\begin{array}{c}\text { Presente } \\
\mathrm{N}=81\end{array}$} & \multirow[t]{2}{*}{ p-valor ${ }^{1}$} & \multicolumn{2}{|c|}{$\begin{array}{l}\text { SI clínica }^{2} \\
N=23\end{array}$} & \multirow[t]{2}{*}{ p-valor 1,3} \\
\hline & $\mathbf{n}$ & $\%$ & $\mathbf{n}$ & $\%$ & $\mathbf{n}$ & $\%$ & & $\mathbf{n}$ & $\%$ & \\
\hline Idade [mediana (IQR)] & 61 & $53 ; 69)$ & $61(5$ & $2 ; 70)$ & $62(4$ & ;71) & 0,800 & 61 & $65)$ & 0,700 \\
\hline Sexo & & & & & & & 0,140 & & & 0,150 \\
\hline Masculino & 93 & 60,0 & 49 & 66,2 & 44 & 54,3 & & 11 & 47,8 & \\
\hline Feminino & 62 & 40,0 & 25 & 33,8 & 37 & 45,7 & & 12 & 52,2 & \\
\hline Raça & & & & & & & 0,400 & & & 0,700 \\
\hline Branca & 71 & 45,8 & 37 & 50,0 & 34 & 42,0 & & 10 & 43,5 & \\
\hline Parda & 70 & 45,2 & 29 & 39,2 & 41 & 50,6 & & 9 & 39,1 & \\
\hline Preta & 14 & 9,0 & 8 & 10,8 & 6 & 7,4 & & 4 & 17,4 & \\
\hline Estadiamento & & & & & & & 0,200 & & & 0,110 \\
\hline I & 6 & 3,9 & 3 & 4,1 & 3 & 3,7 & & 1 & 4,3 & \\
\hline II & 25 & 16,1 & 16 & 21,6 & 9 & 11,1 & & 1 & 4,3 & \\
\hline III & 28 & 18,1 & 12 & 16,2 & 16 & 19,7 & & 4 & 17,4 & \\
\hline IV & 32 & 20,6 & 10 & 13,5 & 22 & 27,2 & & 8 & 34,8 & \\
\hline Não definido & 64 & 41,3 & 33 & 44,6 & 31 & 38,3 & & 9 & 39,2 & \\
\hline Local do Tumor & & & & & & & 0,120 & & & 0,022 \\
\hline Bexiga/rim & 4 & 2,6 & 2 & 2,7 & 2 & 2,5 & & 0 & 0,0 & \\
\hline Cabeça/ tireoide & 18 & 11,6 & 12 & 16,2 & 6 & 7,4 & & 2 & 8,7 & \\
\hline $\begin{array}{l}\text { Cólon/ esôfago/ estômago/ } \\
\text { pâncreas }\end{array}$ & 51 & 32,9 & 23 & 31,1 & 28 & 34,6 & & 4 & 17,4 & \\
\hline Osso/ músculo & 3 & 1,9 & 0 & 0,0 & 3 & 3,7 & & 1 & 4,3 & \\
\hline Útero/ colo útero & 6 & 3,9 & 2 & 2,7 & 4 & 4,9 & & 3 & 13,0 & \\
\hline Hematológico (leucemia/ linfoma) & 28 & 18,1 & 15 & 20,3 & 13 & 16,1 & & 5 & 21,7 & \\
\hline Próstata & 15 & 9,7 & 8 & 10,8 & 7 & 8,6 & & 1 & 4,4 & \\
\hline Pulmão & 6 & 3,9 & 0 & 0,0 & 6 & 7,4 & & 1 & 4,4 & \\
\hline SNC & 5 & 3,2 & 1 & 1,3 & 4 & 4,9 & & 3 & 13,0 & \\
\hline Mama & 10 & 6,4 & 5 & 6,8 & 5 & 6,2 & & 1 & 4,4 & \\
\hline Outros & 9 & 5,8 & 6 & 8,1 & 3 & 3,7 & & 2 & 8,7 & \\
\hline Tratamento & & & & & & & $<0,001$ & & & $<0,001$ \\
\hline Quimioterapia & 37 & 23,9 & 25 & 33,8 & 12 & 14,8 & & 0 & 0,0 & \\
\hline Cirurgia & 65 & 41,9 & 39 & 52,7 & 26 & 32,1 & & 3 & 13,0 & \\
\hline Suporte clínico & 32 & 20,6 & 2 & 2,7 & 30 & 37,0 & & 16 & 69,6 & \\
\hline RT+outros & 8 & 5,2 & 3 & 4,0 & 5 & 6,2 & & 1 & 4,4 & \\
\hline QT+outros & 13 & 8,4 & 5 & 6,8 & 8 & 9,9 & & 3 & 13,0 & \\
\hline Unidade Hospitalar & & & & & & & $<0,001$ & & & $<0,001$ \\
\hline Clínica & 39 & 25,2 & 5 & 6,8 & 34 & 42,0 & & 17 & 73,9 & \\
\hline Cirúrgica & 63 & 40,6 & 39 & 52,7 & 24 & 29,6 & & 1 & 4,4 & \\
\hline de Quimioterapia & 45 & 29,0 & 27 & 36,5 & 18 & 22,2 & & 2 & 8,7 & \\
\hline UTI & 8 & 5,2 & 3 & 4,0 & 5 & 6,2 & & 3 & 13,0 & \\
\hline
\end{tabular}

Nota: ${ }^{1}$ Testes estatísticos realizados: Wilcoxon, para variável Idade e Qui-quadrado via simulação de Monte Carlo para as demais; 'Distribuição da SI clínica (graus 3 a 5) dentre os pacientes com SI presente; ${ }^{3}$ Comparação entre SI clínica (graus 3 a 5) e ausência de SI. Fonte: Vital FMR e Martins CB, 2021. 
A presença de sintomas de moderada a alta intensidade (3-10) como dispneia em repouso, dor, náusea, estresse e fadiga foram indicados pela escala visual analógica nas proporções de aproximadamente $9 \%, 31 \%$, $27 \%, 44 \%$ e $38 \%$, respectivamente, na amostra total de participantes deste estudo.

A presença da SI foi significativamente associada aos sintomas de estresse de alta intensidade $(\mathrm{OR}=5,5$; $[1,96 ; 17,50])$ e fadiga de moderada (OR=3,56; IC95\%=[1,57; 8,58]) e alta intensidade (OR=7,75; $\mathrm{IC} 95 \%=[1,92 ; 52,20])$. Todavia, a presença da SI foi associada apenas a fraqueza muscular (OR=5,17; $I C 95 \%=[1,93 ; 14,60]$ ) dentre sintomas (dispneias, dor, náusea, stress, fadiga) e eventos adversos observados de serem relados no prontuário (pneumonia, atelectasia, insuficiência respiratória, embolia pulmonar, TVP, úlcera de pressão, cálculo renal, anemia, constipação, obstrução intestinal, intolerância à glicose, osteoporose, osteopenia, redução da amplitude de movimento, artralgia, lombalgia, sinais de ansiedade, depressão ou fadiga), embora a fadiga não estivesse presente em nenhum dos pacientes sem SI.

A SI se associou a intensidade e limitação funcional pela fadiga e, também, a deficiência para diversas funções básicas, com exceção da capacidade funcional para realizar trabalho remunerado (Tabela 2). Por exemplo, na Tabela 2, observa-se que um aumento de 1 ponto na CIF para andar distâncias curtas aumenta em seis vezes a chance de um paciente com câncer internado ter SI, assim como o aumento de 1 ponto na CIF para mudar a posição básica do corpo aumenta cerca de quatro vezes a chance de SI.

Tabela 2 - Associação da presença da SI em pacientes com câncer internados com a chance de desenvolver algumas deficiências funcionais e de ter fadiga e o impacto desta na capacidade funcional.

\begin{tabular}{|c|c|c|c|c|c|c|c|c|c|}
\hline \multirow{2}{*}{$\begin{array}{l}\text { Descrição da fadiga e } \\
\text { Função }\end{array}$} & \multicolumn{2}{|c|}{ Total, $\mathrm{N}=155$} & \multicolumn{2}{|c|}{$\begin{array}{c}\text { Ausente, } N= \\
74\end{array}$} & \multicolumn{2}{|c|}{$\begin{array}{c}\text { Presente, } \mathrm{N}= \\
81\end{array}$} & \multirow{2}{*}{$\begin{array}{c}\text { p- } \\
\text { valor }^{1}\end{array}$} & \multirow[t]{2}{*}{ OR } & \multirow[t]{2}{*}{ IC95\% ${ }^{2}$} \\
\hline & Med & IQR & Med & IQR & Med & IQR & & & \\
\hline $\begin{array}{l}\text { Função de mudar a } \\
\text { posição básica do corpo }\end{array}$ & 0 & {$[0,0 ; 2,0]$} & 0 & {$[0,0 ; 0,0]$} & 1 & {$[0,0 ; 3,0]$} & $<0,001$ & 3,9 & {$[2,36 ; 6,49]$} \\
\hline $\begin{array}{l}\text { Função de andar } \\
\text { distâncias curtas }\end{array}$ & 1 & {$[0,0 ; 2,0]$} & 0 & {$[0,0 ; 0,0]$} & 2 & {$[1,0 ; 3,0]$} & $<0,001$ & 6,0 & {$[3,33 ; 10,83]$} \\
\hline $\begin{array}{l}\text { Função de realizar } \\
\text { tarefas domésticas }\end{array}$ & 2 & {$[0,7 ; 4,0]$} & 1 & {$[0,0 ; 2,0]$} & 3 & {$[2,0 ; 4,0]$} & $<0,001$ & 1,8 & {$[1,45 ; 2,27]$} \\
\hline $\begin{array}{l}\text { Função de } \\
\text { relacionamentos sociais } \\
\text { informais }\end{array}$ & 0 & {$[0,0 ; 1,0]$} & 0 & {$[0,0 ; 0,0]$} & 0 & {$[0,0 ; 2,0]$} & $<0,001$ & 1,8 & {$[1,31 ; 2,52]$} \\
\hline $\begin{array}{l}\text { Função de realizar } \\
\text { trabalho remunerado }\end{array}$ & 3 & {$[3,0 ; 3,0]$} & 3 & {$[3,0 ; 3,0]$} & 3 & {$[3,0 ; 3,0]$} & 0,130 & 1,3 & {$[0,98 ; 1,8]$} \\
\hline $\begin{array}{l}\text { Função de executar a } \\
\text { rotina diária }\end{array}$ & 3 & {$[1,0 ; 4,0]$} & 1 & {$[0,0 ; 3,0]$} & 3 & {$[3,0 ; 4,0]$} & $<0,001$ & 2,0 & {$[1,54 ; 2,51]$} \\
\hline $\begin{array}{l}\text { Quanto cansado você se } \\
\text { sentiu na última } \\
\text { semana? }\end{array}$ & 2 & {$[1,0 ; 4,0]$} & 1 & {$[1,0 ; 2,0]$} & 2 & {$[1,0 ; 4,0]$} & $<0,001$ & 1,6 & {$[1,27 ; 2,05]$} \\
\hline $\begin{array}{l}\text { Quanto a sensação de } \\
\text { cansaço de impede de } \\
\text { fazer o que você quer } \\
\text { fazer? }\end{array}$ & 2 & {$[1,0 ; 3,0]$} & 1 & {$[1,0 ; 2,0]$} & 3 & {$[1,0 ; 5,0]$} & $<0,001$ & 1,8 & {$[1,41 ; 2,36]$} \\
\hline
\end{tabular}

Nota: ${ }^{1}$ Teste de Wilcoxon-Mann-Whitney; ${ }^{2}$ Intervalo de confiança de 95\% para OR. Legenda: Med: Mediana; IRQ: Intervalo interquartílico. Fonte: Vital FMR e Martins CB, 2021.

Correlações positivas e significantes foram observadas entre a gravidade progressiva da SI e os eventos adversos fadiga $(r=0,33$; $p$-valor $<0,001)$ e estresse $(r=0,29 ; p$-valor $<0,001)$ mensurados pela EVA, intensidade da fadiga na última semana $(r=0,49 ; p$-valor $<0,001)$ e impacto da fadiga na capacidade funcional na última semana $(r=0,40$; $p$-valor $<0,001)$. As deficiências funcionais: para mudar a posição básica do corpo $(r=0,70 ; p-$ valor $<0,001)$, andar distancias curtas $(r=0,80 ; p$-valor $<0,001)$, realizar tarefas domésticas $(r=0,57 ; p$ valor $<0,001)$, exercer relações sociais informais $(r=0,41 ; p$-valor $<0,001)$ e executar rotina diária $(r=0,55 ; p$ valor $<0,001$ ) também apresentaram correlação positiva significativa com a gravidade da SI (Figura 2). 
Entretanto, não houve correlação estatisticamente significante entre a gravidade da SI e a intensidade dos sintomas dispneia em repouso $(r=0,15 ; p$-valor $=0,061)$, dor $(r=0,08 ; p$-valor $=0,344)$, náusea $(r=0,05 ; p$ valor $=0,545)$ e realizar trabalho remunerado $(r=0,15 ; p$-valor $=0,0614)$.

Figura 2 - Correlação da gravidade progressiva da SI com a progressão da gravidade dos eventos adversos pela EVA, da intensidade e impacto da fadiga pelo Pictograma de fadiga e das deficiências funcionais pela CIF.

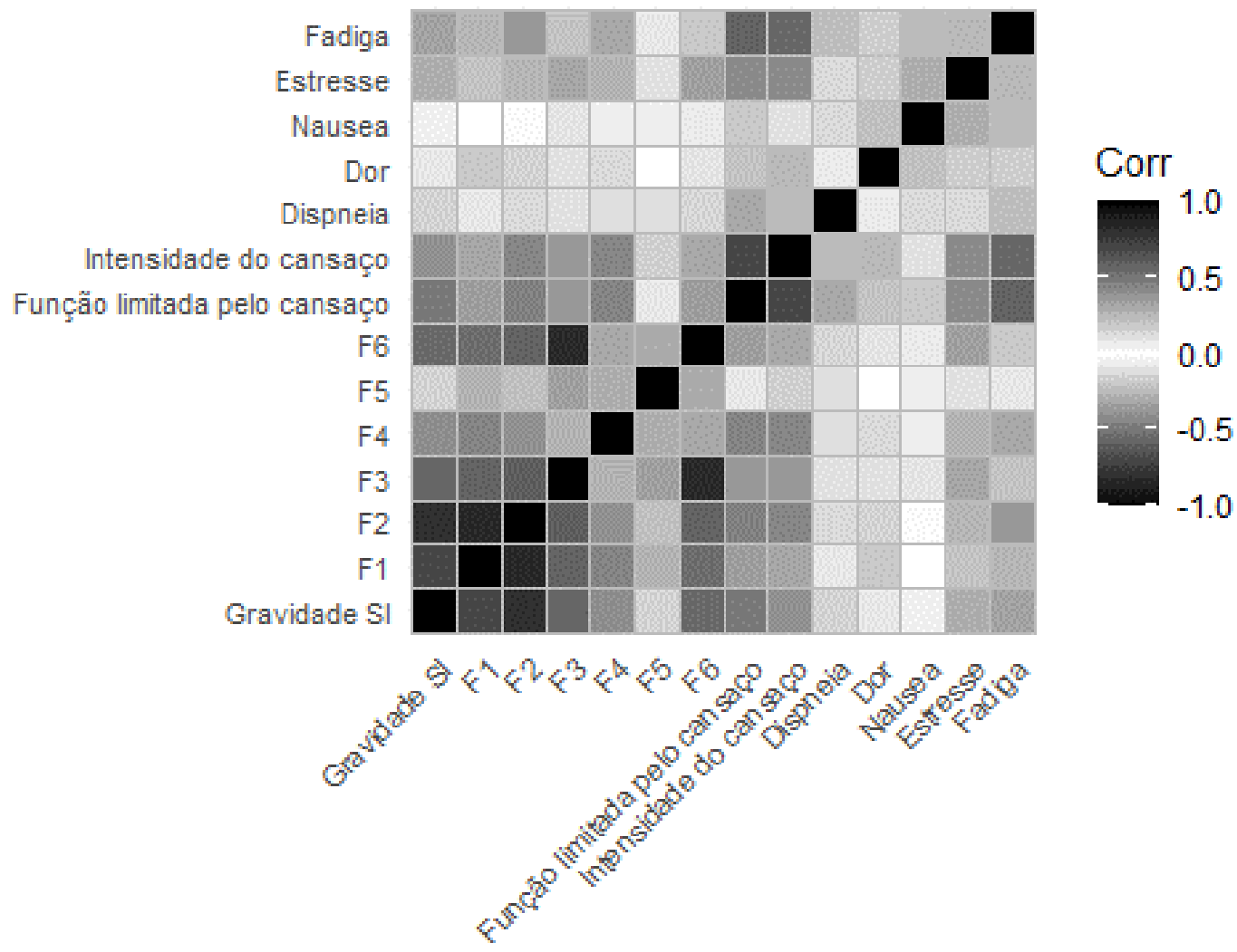

Legenda: $F 1$ = função de mudar a posição básica do corpo; F2= função de andar distâncias curtas; F3= função de realizar tarefas domésticas; $F 4=$ função de relacionamentos sociais informais; $F 5=$ função de realizar trabalho remunerado; F6= função de executar a rotina diária. Fonte: Vital FMR e Martins CB, 2021.

\section{DISCUSSÃO}

Foi demonstrado que a SI tem uma prevalência moderada (52\%) em pacientes com câncer internados, podendo estar presente em até $15 \%$ destes quando tem gravidade clinicamente relevante. Embora em termos percentuais não pareça muito, este valor equivale a estimativa de 119 mil brasileiros portadores de câncer que em um ano estarão com uma limitação clinicamente relevante da sua mobilidade durante uma internação, o que foi associado a presença de sintomatologias, fraqueza e perdas funcionais, o que denota os impactos clínicos desta síndrome e aponta os possíveis impactos econômicos com internações mais prolongadas, reinternações e dias de trabalho perdidos (CAIXETA NETO, et al., 2019).

A SI esteve mais presente no perfil de pacientes com câncer internados para suporte clínico em unidades clínicas e a redução da mobilidade, clinicamente relevante, esteve presente, em especial, nos pacientes com diagnóstico de câncer no SNC, pulmão, musculoesquelético e útero. No entanto, muitas vezes, este perfil é negligenciado de ser encaminhado para fisioterapia durante uma internação hospitalar. Todos os pacientes cirúrgicos da unidade de internação cirúrgica da instituição onde este estudo foi conduzido recebiam assistência diária de fisioterapia no modelo do projeto ERAS, que se resume em um plano de cuidados multidisciplinar, onde protocolos são auditados quanto a aderência das equipes, talvez por isso não tenha sido identificada a presença da SI de forma significativa nesta população investigada (LJUNGQVIST O, et al., 2017). 
A nova perspectiva que o ERAS validou, ao estimular a mobilização precoce junto a padronização de outras ações preventivas multidisciplinares ocorreu literalmente na Europa, quando cirurgiões da academia europeia se reuniram para rastrear a literatura em busca das intervenções que mais contribuíam para acelerar a recuperação no pós-operatório de colectomias e identificaram que a mobilização precoce era um importante fator e estruturaram o reconhecido projeto (LJUNGQVIST O, et al., 2017). A aplicação desses protocolos pode levar a uma redução de 30-50\% das complicações pós-operatórias, do tempo de permanência hospitalar e, ainda, uma redução das readmissões e nos custos hospitalares (BOND-SMITH G, et al., 2016).

A fraqueza e a pobre capacidade funcional são fortes preditores de readmissão hospitalar e morte. A frequência de readmissões após cirurgias oncológicas de grande porte varia de $16 \%$ a $25 \%$ em até 30 dias e de $53 \%$ a $66 \%$ em até um ano. Alguns fatores de risco que veem sendo identificados como preditivos de readmissão nesta população são: idade; comorbidades, tempo de permanência hospitalar; condição préoperatória, em especial fraqueza e redução do status performance funcional, ou seja, muitas readmissões são preveníveis (ROCHEFORT MM e TOMLINSON JS, 2012).

Atualmente, há vasta literatura demonstrando que a mobilização, durante a internação, traz semelhantes ou mais benefícios para os pacientes com ou em risco de disfunções respiratórias (ZHANG L, et al., 2019; FOSSAT G, et al., 2018; CONNOLLY B, et al., 2016). Podemos exemplificar com a gama de literatura que demonstra a redução na incidência de pneumonia em pacientes em pós-operatório de cirurgias de grande porte quando estimulados a deambular precocemente comparado ao tratamento médico padrão ou mesmo a só fisioterapia respiratória no pós-operatório (BHATT NR, et al., 2017; BOND-SMITH G, et al., 2016; SILVA YR, et al., 2013).

A SI clinicamente relevante foi associada a presença de alta intensidade de estresse e moderada a alta intensidade de fadiga na percepção dos participantes do estudo. Todavia o evento fadiga não foi encontrado em nenhum dos registros de prontuário ou pelo relato do paciente em quem não desenvolveu a SI durante a internação, o que poderia estar relacionado a falha no registro e poderia indicar a hipótese de que a redução da mobilidade é um importante fator de risco para o desenvolvimento de fadiga, ou o oposto.

Foi possível demonstrar, também, que a percepção de intensidade de fadiga e as limitações funcionais relacionadas a ela se associam a uma maior chance de ter SI, assim como a redução da capacidade de realizar funções básicas como realizar trabalhos domésticos, executar a rotina diária e as relações sociais informais estão associadas a uma maior chance de ter SI. Assim como uma correlação progressiva da gravidade da SI com a progressão da intensidade dos sintomas fadiga e estresse e, ainda, perdas funcionais avaliadas. Nós não identificamos a exploração dessas associações em nenhum outro estudo em pacientes com câncer.

Considerando o tamanho da amostra incluído e o desenho de estudo transversal, não foi possível observar a presença ou associação de muitos dos eventos adversos que podem ser consequência da redução da mobilidade como descritos na literatura (CAO J, et al., 2021; GUEDES LPCM, et al., 2018). A falta de registro em prontuário do estadiamento da doença e eventos adversos ocorridos durante a internação ainda é fato comum, embora inadequado, e podem ter comprometido a identificação da associação com a SI.

Pacientes admitidos para uma internação hospitalar ou com prognóstico para internação maior que de 3 dias, devem ser triados em busca de sinais e sintomas da SI e serem estimulados a mobilizar para, assim, evitar eventos adversos e perdas funcionais que podem ter importante impacto social e econômico, considerando que uma alta hospitalar com perdas funcionais pode levar a necessidade de reabilitação por tempo prolongado, reinternações e atraso do retorno ao trabalho. Adicionalmente, as perdas funcionais consequentes ou associadas a SI podem impactar na aderência ao tratamento oncológico, na qualidade de vida e na sobrevida.

Contudo, há dificuldade de se obter o reembolso para realizar o rastreamento de pacientes internados com câncer que poderiam necessitar de reabilitação. Portanto, são necessárias estratégias baseadas em fatores de risco para complicações médicas, como SI, fadiga, estresses, fraqueza, além de uma melhor comunicação e coordenação com as equipes de oncologia (FU JB, et al., 2017). 


\section{CONCLUSÃO}

Este estudo indica que a SI está presente em metade dos pacientes internados com câncer. Uma gravidade clínica relevante desta síndrome pode acometer cerca de 15\% desta população. Esta síndrome está associada a pacientes que também apresentam estresse, fadiga e fraqueza muscular. Quanto maior a gravidade da SI, mais intensa tende a ser a fadiga, o estresse, o impacto da fadiga nas AVDs e também na capacidade funcional. Portanto são necessárias ações preventivas ou terapêuticas precoces para minimizar os possíveis impactos clínicos, sociais, econômicos, no tratamento oncológico e na qualidade de vida dos pacientes com esta síndrome.

\section{AGRADECIMENTOS}

Agradecemos a Cristiane Aparecida de Faria, Izabel Carvalho Ferreira e Kássia Elisa Vardiero pela colaboração na coleta dos dados e a Fundação Cristiano Varella pela concessão em executarmos a coleta de dados junto aos pacientes da instituição.

\section{REFERÊNCIAS}

1. BHATT NR, et al. Postoperative exercise training is associated with reduced respiratory infection rates and early discharge: A case-control study. Surgeon. 2017; 15(3): 139-46.

2. BOND-SMITH G, et al. Enhanced recovery protocols for major upper gastrointestinal, liver and pancreatic surgery. Cochrane Database Syst Rev. 2016; 2: CD011382.

3. CAIXETA NETO, et al. Braz. Perfil nutricional de pacientes oncológicos internados na Santa Casa de Misericórdia de Ouro Preto - MG. J. Surg. Clin. Res.2019; 28(2): 08-10.

4. CAO J, et al. Risk factors associated with deep venous thrombosis in patients with different bed-rest durations: A multiinstitutional case-control study. Int J Nurs Stud. 2021;114: 103825.

5. CHEN S, et al. Physical training is beneficial to functional status and survival in patients with prolonged mechanical ventilation. J Formos Med Assoc. 2011; 110(9): 572-9.

6. CONNOLLY B, et al. Physical rehabilitation interventions for adult patients during critical illness: an overview of systematic reviews. Thorax. 2016; 71(10): 881-90.

7. ESPÍNDOLA R, et al. Prevalência de cuidados paliativos em pacientes com síndrome de imobilidade em um serviço de atenção domiciliar. Semina cienc. biol. Saúde. 2020; 41(2): 229-238.

8. FOSSAT G, et al. Effect of In-Bed Leg Cycling and Electrical Stimulation of the Quadriceps on Global Muscle Strength in Critically III Adults: A Randomized. Clinical Trial. JAMA. 2018; 320(4): 368-378.

9. FU JB, et al. A Guide to Inpatient Cancer Rehabilitation: Focusing on Patient Selection and Evidence-Based Outcomes. PM R. 2017; 9(9S2): S324-S334.

10. GARCIA-PEREZ L, et al. Risk factors for hospital readmissions in elderly patients: a systematic review. QJM. 2011; 104(8): 639-51.

11. GUEDES LPCM, et al. Deleterious effects of prolonged bed rest on the body systems of the elderly - a review. Rev. bras. geriatr. gerontol. 2018; 21(4): 499-506.

12. GUO $\mathrm{Y}$, et al. Inpatient rehabilitation improved functional status in asthenic patients with solid and hematologic malignancies. Am J Phys Med Rehabil. 2011; 90(4): 265-71.

13. HAJJAR LA, et al. Diretriz Brasileira de Cardio-oncologia - 2020. Arq Bras Cardiol. 2020; 115(5): 1006-1043.

14. HERMANS G, et al. Acute outcomes and 1-year mortality of intensive care unit-acquired weakness. A cohort study and propensity-matched analysis. Am J Respir Crit Care Med. 2014; 190(4): 410-20.

15. IZCOVICH A, et al. Early mobilization versus bed rest for deep vein thrombosis. Medwave. 2016;16 Suppl 2: e6478.

16. KALIL FILHO R, et al. I Brazilian Guideline for Cardio-Oncology from Sociedade Brasileira de Cardiologia. Arq Bras Cardiol. 2011; 96(2 Suppl 1): 1-52.

17. KANG SH, et al. Multimodal Enhanced Recovery After Surgery (ERAS) Program is the Optimal Perioperative Care in Patients Undergoing Totally Laparoscopic Distal Gastrectomy for Gastric Cancer: A Prospective, Randomized, Clinical Trial. Ann Surg Oncol. 2018; 25(11): 3231-8.

18. KEHLER DS, et al. Bed rest and accelerated aging in relation to the musculoskeletal and cardiovascular systems and frailty biomarkers: A review. Exp Gerontol. 2019; 124: 110643.

19. LIU Z, et al. Bed rest versus early ambulation with standard anticoagulation in the management of deep vein thrombosis: a meta-analysis. PLoS One. 2015; 10(4):e0121388.

20. LJUNGQVIST O, et al. Enhanced Recovery After Surgery: A Review. JAMA Surg. 2017; 152(3): 292-8. 
21. LUM HD, et al. Early hospital readmission is a predictor of one-year mortality in community-dwelling older Medicare beneficiaries. J Gen Intern Med. 2012; 27(11): 1467-74.

22. MACHADO ADS, et al. Effects that passive cycling exercise have on muscle strength, duration of mechanical ventilation, and length of hospital stay in critically ill patients: a randomized clinical trial. J Bras Pneumol. 2017; 43(2): 134-139.

23. MOTA DDCF, et al. Fatigue Pictogram: an option for assessing fatigue severity and impact. Rev. Esc. Enferm. USP. 2009; 43: 1080-1087.

24. ORGANIZAÇÃO MUNDIAL DA SAÚDE (OMS). CIF: Classificação Internacional de Funcionalidade, Incapacidade e Saúde. Trad. do Centro Colaborador da Organização Mundial da Saúde para a Família de Classificações Internacionais. São Paulo: EDUSP; 2003.

25. PLAPLER PG, et al. Relationship between the coronavirus disease 2019 pandemic and immobilization syndrome. Clinics. 2021; 76: e2652.

26. ROCHEFORT MM, TOMLINSON JS. Unexpected readmissions after major cancer surgery: an evaluation of readmissions as a quality-of-care indicator. Surg Oncol Clin N Am. 2012; 21(3): 397-405.

27. SANTOS C, et al. Mechanisms of Chronic Muscle Wasting and Dysfunction after an Intensive Care Unit Stay. A Pilot Study. Am J Respir Crit Care Med. 2016; 194(7): 821-30.

28. SAUNDERS CB. Preventing secondary complications in trauma patients with implementation of a multidisciplinary mobilization team. J Trauma Nurs. 2015; 22(3): 170-5.

29. SILVER JK, Baima J, Mayer RS. Impairment-driven cancer rehabilitation: an essential component of quality care and survivorship. CA Cancer J Clin. 2013; 63(5):295-317.

30. SHIDA D, et al. Modified enhanced recovery after surgery (ERAS) protocols for patients with obstructive colorectal cancer. BMC Surg. 2017; 17(1): 18.

31. SILVA YR, et al. Does the addition of deep breathing exercises to physiotherapy-directed early mobilisation alter patient outcomes following high-risk open upper abdominal surgery? Cluster randomised controlled trial. Physiotherapy. 2013; 99(3): 187-93.

32. VITAL FMR. Fisioterapia em Oncologia: protocolos assistenciais. Rio de Janeiro: Atheneu; 2017; 461p.

33. ZHANG L, et al. Early mobilization of critically ill patients in the intensive care unit: A systematic review and metaanalysis. PLoS One. 2019; 14(10): e0223185. 